\title{
A Multi-Soft-Body Dynamic Model for Underwater Soft Robots
}

\author{
Federico Renda, Francesco Giorgio-Serchi, Frederic Boyer, Cecilia Laschi, Jorge \\ Dias and Lakmal Seneviratne.
}

\begin{abstract}
We present a unified formulation for describing the dynamics of a new class of aquatic multi-body soft robots. This formulation accounts for the continuum hyperelastic nature of the vehicles and for the interaction with the dense fluid across which they dwell. We start by introducing the highly unconventional design concept of a soft underwater vehicle inspired by the octopus capable of swimming, manipulating and crawling. The dynamics of the robot, which consists of a multi-limbed continuum of elastomeric materials, is extremely complex to account for with conventional modelling tools. Hence a Cosserat based formalism where a Reissner shell model and a finite-strain beam formulation are joined is conceived which lends itself to the description of the highly non linear dynamics of this new family of vehicles in a dense fluid.
\end{abstract}

\section{Introduction}

Marine operations and the growing needs of the offshore industry require underwater robots to undertake increasingly daunting tasks in always more forbidding environments. Certain scenarios, however, pose such challenges at sea that standard Remotely Operated Vehicles (ROVs) and Autonomous Underwater Vehicles (AUVs) are likely to be unsuitable. An answer to this problem lies in the development of in-

F. Renda, J. Dias, L. Seneviratne

KURI, Khalifa University, Abu Dhabi, UAE.

e-mail: federico.renda@kustar.ac.ae; jorge.dias@kustar.ac.ae; lakmal.seneviratne@kustar.ac.ae

F. Giorgio Serchi, C. Laschi

the BioRobotics Institute, Scuola Superiore Sant'Anna, Pisa, Italy. e-mail: f.serchi@sssup.it; cecilia.laschi@sssup.it

F. Boyer

IRCCyN, Ecole de Mines de Nantes, Nantes, France. e-mail: frederic.boyer@mines-nantes.fr 
novative underwater robots which, endowed with augmented manoeuvrability and flexibility, may provide an aid to the ever growing demands of the marine sector.

In recent times underwater robotics has largely benefited from the growing fascination for bioinspired locomotion, because the design of underwater robots can profit massively from the investigation of the swimming strategies, hydrodynamics and physiology of such animals. Several examples exist of aquatic organisms which have been taken as the source of inspiration for designing a trustworthy robotic counterpart. The finned and caudal flapping of fish (e.g. [1]) has gathered the most recognition in the scientific community, in part because of the sound understanding of the underlying physics involved in their locomotion [2].

The design criteria for replicating the actuation mechanism which drives the propulsion of fish has, in most cases, entailed the replacement of continuously deforming bodies by reducing the number of degrees-of-freedom (DOF) with a finite sequence of rigid links and joints. However, lately the attempt has been made by [3] to account for the compliant nature of these organisms by resorting to continuous soft structures and actuators. This is one of the few examples where the design principles of soft robotics [4] have been adapted to the aquatic context. In water the hindrances due to the lack of rigid parts are compensated by the support of the dense medium in which the vehicle is immersed, annihilating many of the limits which soft robots are faced with on land. This has encouraged the authors to develop a new breed of aquatic soft robots inspired by the quintessential soft-bodied sea dweller, the octopus.

While the design of Soft Unmanned Underwater Vehicles (SUUVs), may result fairly uncomplicated, their modeling and control is anything but straight forward. Here we present the first example of a cable-actuated, multi-body, aquatic soft robot and introduce a formulation which accounts for the continuum nature of the robotic platform and allows to describe the dynamics of this vehicle while it travels in a quiescent fluid.

\subsection{An aquatic multi-body soft robot}

The octopus sports a range of features which are very much sought for in underwater robotics. These include essentially the capability to swim, crawl and manipulate along with an overall remarkable structural compliance. These make the octopus the perfect paradigm of aquatic vehicle. In the scenario of offshore intervention, where complex environments and highly perturbed conditions are the norm, the design criteria borrowed from the soft/bioinspired approach could represent a viable solution to a broad range of tasks which current Unmanned Underwater Vehicles (UUVs) are unfit for.

By taking inspiration from the octopus, the authors have developed a soft-bodied vehicle capable of travelling in water and replicating some of the salient skills of this aquatic organism [5]. The result of this effort is portrayed schematically in Fig. 1. This first prototype of aquatic multi-body soft robot entails a platform composed 
Fig. 1 A schematic of the SUUV developed by the authors. Numbers refer to: (1) pulsed-jet thruster, (2) the nozzle, (3) the cables which drive the shell collapse, (4) the continuum manipulators, (5) the actutors of the manipulators, (6) the actuator of the shell and (7) the cable which drives manipulator actuation.

Fig. 2 The Soft Unmanned Underwater Vehicle (SUUV) PoseiDRONE upon assemblage completion (a) and during testing at sea (b).
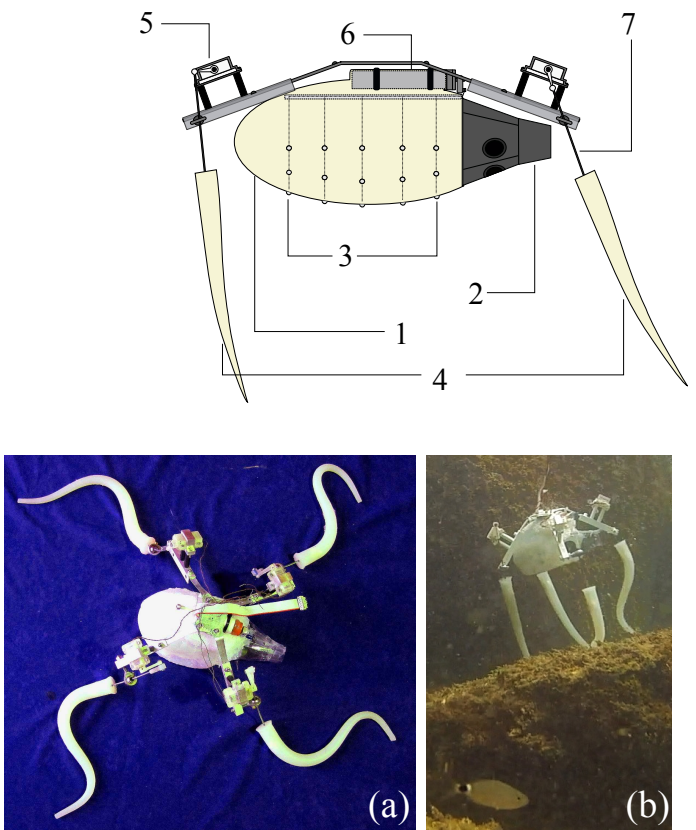

for as much as $80 \%$ in volume of rubber-like materials and capable of manipulating as well as travelling in the aquatic environment either via waterborne pulsed-jet propulsion or legged locomotion.

The robot essentially consist of a central elastic shell, referred to here as the Soft Shell Mantle (SSM), which is designated to performing the pulsed-jet propulsion via the recursive ingestion and expulsion of finite slugs of ambient water [6, 7]. From this central unit, a number of manipulators, i.e. the Soft Robot Arms (SRAs), depart: these are conical-shaped continuous structures composed of elastomeric materials designated to performing basic manipulation [8] and legged-locomotion [9]. Actuation is entirely dealt with via cable-transmission: inextensible cables run through the arms and inside the central shell and, upon recoiling from the designated DC motor, drive the twirling of the manipulator or the collapse of the shell.

Once assembled, the vehicles appears as in Fig. 2(a). This vehicle has been tested both in controlled environments as well as in open water, see Fig. 2(b) and modeling and control of this complex system has been attempted by separately accounting for the various mechanical units $[6,10]$. Here, for the first time, the authors attempt to formulate a unified model which encompasses internal actuation and external dynamics. 


\section{Soft Robot Arm and Soft Shell Mantle Model}

In the geometrical exact approach, the SRA is viewed as a Cosserat rod [11], i.e. as a continuous assembly of rigid cross section, while the SSM is modelled as a Cosserat axisymetric shell [12], i.e. as a continuous assembly of fibers along the median surface. In this section, a brief description of the kinematics and dynamics of a Cosserat rod/shell for underwater soft robotics is given, based on the authors previous works [8], [13], which should be taken as the reference for a more detailed derivation. Experimental validation of the model are also presented in [8] for the SRA, while for the SSM steady state experiments have been presented in [14], dynamic experiments are under review and a coupled dynamic-potential flow solution is given in [15]. In order to appreciate the symmetry between the two models, with a slight abuse of notation, some times we will adopt the same symbols for the two formulations, since they share the same geometrical and mechanical meaning in both the cases.

\subsection{Kinematics}

The reference space is endowed with a base of orthogonal unit vectors $\left(\mathbf{e}_{1}, \mathbf{e}_{2}, \mathbf{e}_{3}\right)$ (Fig. 3). In the Cosserat theory, the configuration of a soft body at a certain time is characterized by a position vector $\mathbf{r}$ and a material orientation matrix $R$, parameterized by the material abscissas, that are $\phi \in[0,2 \pi[$, the angle of revolution of the axisymmetric surface, and $X \in\left[0, L_{S}\right]$ the abscissa along the meridian for the SSM; and $X \in\left[0, L_{b}\right]$, the abscissa along the robot arm, for the SRA (the subscripts $s$ and ${ }_{b}$ stand respectively for shell and beam). Thus, the configuration space is defined as a curves $g_{b}(X)$ and a surface $g_{s}(X, \phi) \in S E(3)$, with $g_{b}=\left(\begin{array}{cc}R_{b} & \mathbf{r}_{b} \\ 0 & 1\end{array}\right)$ and $g_{s}=\left(\begin{array}{cc}R_{s} & \mathbf{r}_{\mathbf{s}} \\ 0 & 1\end{array}\right)$

In order to exploit the axisymmetry of the SSM, we introduce another orthogonal basis attached to the material point $(X, \phi):\left(\mathbf{e}_{r}, \mathbf{e}_{\phi}, \mathbf{e}_{3}\right)$ (Fig. 3); defined by rototraslating $\left(\mathbf{e}_{1}, \mathbf{e}_{2}, \mathbf{e}_{3}\right)$ of $g_{1}(X, \phi)$ equal to: $g_{1}=\left(\begin{array}{cc}\exp \left(\tilde{\mathbf{e}}_{3} \phi\right) & \mathbf{r}_{s} \\ 0 & 1\end{array}\right)$, where exp is the exponential in $\mathrm{SO}(3)$. In this case $\mathbf{r}_{s}(X)$ take the form: $\mathbf{r}_{s}=(\cos (\phi) r, \sin (\phi) r, z)^{T}$ for which, $r(X)$ and $z(X)$ are two smooth functions which define the radius and the altitude of the point $X$ on the profile (Fig. 3). For the sake of convenience, we introduce another orthogonal basis $\left(\mathbf{e}_{r}, \mathbf{e}_{3},-\mathbf{e}_{\phi}\right)$ rotated from the former by $g_{2}=\left(\begin{array}{cc}\exp \left(\tilde{\mathbf{e}}_{r} \pi / 2\right) & 0 \\ 0 & 1\end{array}\right)$. Then, if we call $\theta(X)$ the angle between $\mathbf{e}_{3}$ and the shell fiber located at any $X$ along the $\phi$-meridian, the so called director orthogonal frame $(\mathbf{x}, \mathbf{y}, \mathbf{z})$ is defined at each instant $t$, by $g_{3}(X)$ equal to: $g_{3}=\left(\begin{array}{cc}\exp \left(-\tilde{\mathbf{e}}_{\phi} \theta\right) & 0 \\ 0 & 1\end{array}\right)$. Finally, putting them all together, the shell configuration space is $g_{s}=g_{1} g_{2} g_{3}$. 
Fig. 3 Sketch of the kinematics which show the geometrical meaning of the elements $g, \xi$ and $\eta$. The reference frames on the figure are those used in the model.

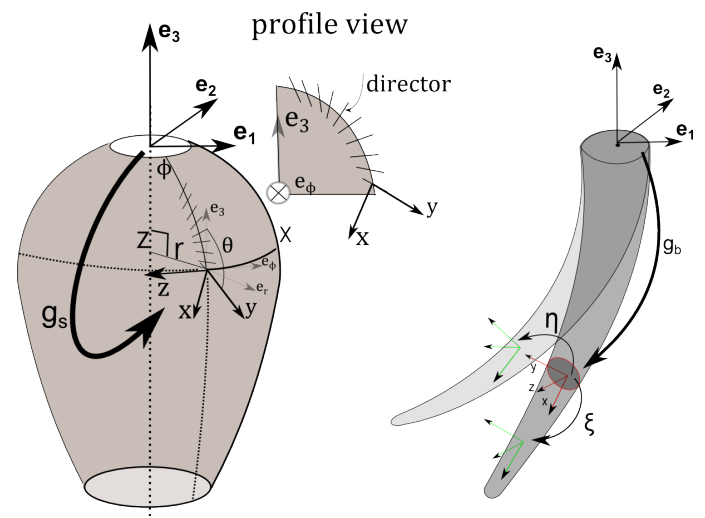

Now, the tangent vector field along the curve $g_{b}(X)$ is defined by $\widehat{\xi}(X)=$ $g_{b}^{-1} \partial g_{b} / \partial X=g_{b}^{-1} g_{b}^{\prime} \in s e(3)$ and the tangent plane on the surface $g_{s}(X, \phi)$ is defined by the two vector fields: $\widehat{\xi}_{1}(X)=g_{s}^{-1} \partial g_{s} / \partial X=g_{s}^{-1} g_{s}^{\prime}$ and $\widehat{\xi}_{2}(X)=g_{s}^{-1} \partial g_{s} / \partial\left(r^{\star} \phi\right)=$ $g_{s}^{-1} g_{s}^{2}$ (where ${ }^{\star}$ denote variable in the reference configuration). In local frame components we have: $\xi=\left(\mathrm{k}^{T}, \mathrm{~g}^{T}\right)^{T}, \xi_{1}=\left(\mathrm{k}_{1}^{T}, \mathrm{~g}_{1}^{T}\right)^{T}=(0,0, \mu, \lambda, \beta, 0)^{T}, \xi_{2}=$ $\left(\mathrm{k}_{2}^{T}, \mathrm{~g}_{2}^{T}\right)^{T}=\left(\frac{\sin (\theta)}{r^{\star}}, \frac{\cos (\theta)}{r^{\star}}, 0,0,0,-\frac{r}{r^{\star}}\right)^{T} \in \mathbb{R}^{6}$. where $\mathrm{g}(X)$ represents the linear strains, and $\mathrm{k}(X)$ the angular strain. The hat is the isomorphism between the twist vector space $\mathbb{R}^{6}$ and the Lie algebra $s e(3)$.

The time evolution of the configuration curve $g_{b}$ and surface $g_{s}$ is represented by the twist vector field $\eta(X) \in \mathbb{R}^{6}$ defined respectively by $\widehat{\eta}_{b}=g_{b}^{-1} \partial g_{b} / \partial t=g_{b}^{-1} g_{b}$ and $\widehat{\eta}_{s}=g_{s}^{-1} \partial g_{s} / \partial t=g_{s}^{-1} \dot{g}_{s}$. As before, in the local components we have: $\eta_{b}=$ $\left(\mathrm{w}_{b}^{T}, \mathrm{v}_{b}^{T}\right)^{T}, \eta_{s}=\left(\mathrm{w}_{s}^{T}, \mathrm{v}_{s}^{T}\right)^{T}=\left(0,0, \Omega, V_{x}, V_{y}, 0\right)^{T} \in \mathbb{R}^{6}$, where $\mathrm{v}(X)$ and $\mathrm{w}(X)$ are respectively the linear and angular velocity of a material element at a given instant.

In accordance with this kinematics, the state vector of the SRA and of the SSM are represented by the terns $\left(g_{b}, \xi, \eta_{b}\right)$ and $\left(g_{s}, \xi_{1}, \eta_{s}\right)$. From the development above, we can derived the kinematic equations (1) and (2), while in the next sections the compatibility equations and the dynamic equations will be derived (the tilde is the isomorphism between a vector of $\mathbb{R}^{3}$ and the corresponding skew-symmetric matrix $\in \operatorname{so}(3))$.

$$
\begin{gathered}
\dot{\mathbf{r}}_{b}=R_{b} \mathrm{v}_{b} \quad \dot{R}_{b}=R_{b} \widetilde{\mathrm{w}}_{b} \\
\dot{\theta}=\Omega \\
\dot{r}=\cos (\theta) V_{x}-\sin (\theta) V_{y} \\
\dot{z}=\sin (\theta) V_{x}+\cos (\theta) V_{y}
\end{gathered}
$$

\subsection{Strain Measures}

There are different ways to measure the strain of a continuous media, we choose the most common used in the specialized literature for the beam and shell separately. 
For the SRA, the strains are defined as the difference between the deformed configuration $\xi$ and the reference configuration $\xi^{\star}$. In particular, the components of $k-k^{\star}$ measure the torsion and the bending state in the two directions. Similarly, the components of $g-g^{\star}$ represent the longitudinal strain (extension, compression) and the two shear strains.

For the SSM, in accordance with [12] as described in [13], the strain tensor field which describes the membrane strain state in the mid-surface is $e(X)=$ $1 / 2\left(h-h^{\star}\right)$ where $h(X)$ is the first fundamental form of the Reissner shell equal to $h=\operatorname{diag}\left(\lambda^{2}+\beta^{2}, r^{2} / r^{\star 2}\right)$. Thus we have $e=(1 / 2) * \operatorname{diag}\left(\lambda^{2}+\beta^{2}-1, r^{2} / r^{\star 2}-1\right)$, in which we have defined $h_{11}^{\star}=1$. For what concerns the shear strain state, we have $s(X)=\beta-\beta^{\star}$. Finally, the flexural strain state is parametrized by the tensor field $d(X)=k-k^{\star}$, where $k(X)$ is the second fundamental form equal to $k=\operatorname{diag}\left(-\mu \lambda,-r \sin (\theta) / r^{\star 2}\right)$. Thus we have $d=\operatorname{diag}\left(\mu^{\star}-\mu \lambda, \sin \left(\theta^{\star}\right) / r^{\star}-\right.$ $\left.r \sin (\theta) / r^{\star 2}\right)$. Furthermore, it is natural to consider that there is no transverse shearing in the reference resting configuration, i.e. $\beta^{\star}=0$.

\subsection{Compatibility Equations}

We have seen above that $g_{b}^{\prime}=g_{b} \widehat{\xi}$ and $g_{s}^{\prime}=g_{s} \widehat{\xi}_{1}$. By taking the derivative of these equations with respect to time and recalling that $\dot{g}=g \widehat{\eta}$, we obtain the following compatibility equations between velocity and deformation variables: $\dot{\xi}=$ $\eta_{b}^{\prime}+\operatorname{ad}_{\xi}\left(\eta_{b}\right)$ and $\dot{\xi}_{1}=\eta_{s}^{\prime}+\operatorname{ad}_{\xi_{1}}\left(\eta_{s}\right)$, where ad is the adjoint map. In local components, we obtain:

$$
\begin{aligned}
\dot{\mathrm{g}} & =\mathrm{v}_{b}^{\prime}+\mathrm{k} \times \mathrm{v}_{b}-\mathrm{w}_{b} \times \mathrm{g} \\
\mathrm{\textrm {k }} & =\mathrm{w}_{b}^{\prime}+\mathrm{k} \times \mathrm{w}_{b} \\
\dot{\mu} & =\Omega^{\prime} \\
\dot{\lambda} & =V_{x}^{\prime}+\beta \Omega-\mu V_{y} \\
\dot{\beta} & =V_{y}^{\prime}-\lambda \Omega+\mu V_{x}
\end{aligned}
$$

\subsection{Dynamics}

The p.d.e.'s describing the evolution of a Reinsner rod and shell (not necessarily axisymmetric) have been derived respectively in [11] and [12]. With respect to the local reference frame, these p.d.e's can be written, in a geometric notation, as: $\mathscr{M}_{b} \dot{\eta}_{b}=\mathscr{F}_{b i}^{\prime}+\operatorname{ad}_{\xi}^{*}\left(\mathscr{F}_{b i}\right)+\mathscr{F}_{b e}-\operatorname{ad}_{\eta_{b}}^{*}\left(\mathscr{M}_{b} \eta_{b}\right)$ and $\mathscr{M}_{s} \dot{\eta}_{s}=1 / j\left(j \mathscr{F}_{s i}^{1}\right)^{\prime}+$ $\operatorname{ad}_{\xi_{\alpha}}^{*}\left(\mathscr{F}_{s i}^{\alpha}\right)+\mathscr{F}_{s e}-\operatorname{ad}_{\eta_{s}}^{*}\left(\mathscr{M}_{s} \eta_{s}\right)$, where $j=\sqrt{\operatorname{det}(h)}=r / r^{\star} \sqrt{\lambda^{2}+\beta^{2}}, \mathscr{F}_{b i}(X)$ and $\mathscr{F}_{s i}^{\alpha}(X)$ are the wrenches of internal forces in the surface directions given by $g_{\alpha}(\alpha$ running over $\{1,2\}), \mathscr{F}_{b e}(X)$ and $\mathscr{F}_{s e}(X)$ are the external wrench of distributed applied forces, $\mathscr{M}_{b}(X)$ and $\mathscr{M}_{s}(X)$ are the screw inertia matrix and $\mathrm{ad}^{*}=-\mathrm{ad}^{T}$ is the co-adjoint map. For the repeated $\alpha$ the Einstein convention has 
to be used as in the rest of the paper. Let us specify the angular and linear components of the internal and external wrenches respectively ( for the axisymmetric case see [16]): $\mathscr{F}_{b i}=\left(\mathrm{M}_{b}^{T}, \mathrm{~N}_{b}^{T}\right)^{T}, \mathscr{F}_{s i}^{1}=\left(\mathrm{M}_{s}^{1 T}, \mathrm{~N}_{s}^{1 T}\right)^{T}=\left(0,0, M_{X}, N_{X}, H, 0\right)^{T}$, $\mathscr{F}_{s i}^{2}=\left(\mathrm{M}_{s}^{2 T}, \mathrm{~N}_{s}^{2 T}\right)^{T}=\left(M_{\phi_{x}}, M_{\phi_{y}}, 0,0,0,-N_{\phi}\right)^{T} \in \mathbb{R}^{6}$, and $\mathscr{F}_{b e}=\left(\mathrm{m}_{b}^{T} \mathrm{n}_{b}^{T}\right)^{T}, \mathscr{F}_{s e}=$ $\left(\mathrm{m}_{s}^{T}, \mathrm{n}_{s}^{T}\right)^{T}=\left(0,0, l, f_{x}, f_{y}, 0\right)^{T} \in \mathbb{R}^{6}$, where $\mathrm{N}(X)$ and $\mathrm{M}(X)$ are the internal force and torque vectors, respectively, while $\mathrm{n}(X)$ and $\mathrm{m}(X)$ are the external force and torque for unit of $X$. The screw inertia matrices are equal to: $\mathscr{M}_{b}=\rho_{b} *$ $\operatorname{diag}\left(I_{b}, J_{b}, J_{b}, A, A, A\right)$ and $\mathscr{M}_{s}=\rho_{s} * \operatorname{diag}\left(J_{s}, I_{s}, J_{s}, 2 h_{s}, 2 h_{s}, 2 h_{s}\right) \in \mathbb{R}^{6} \times \mathbb{R}^{6}$. In the equations above $\rho_{b}$ and $\rho_{s}$ are the body densities, $A(X)$ is the section area equal to $A=\pi h_{b}^{2}$, where $h_{b}(X)$ is the cross section radius, $h_{s}$ is the half of the shell thickness and $J(X), I(X)$ are the second moment of inertia of the micro-solid equal to $J_{b}=\pi h_{b}^{4} / 4, J_{s}=h_{s}^{2} / 3, I_{b}=\pi h_{b}^{4} / 2, I_{s} \sim 0$. In components, the dynamic equations are:

$$
\begin{gathered}
\rho_{b} A \dot{\mathrm{v}}_{b}=\mathrm{N}_{b}^{\prime}+\mathrm{k} \times \mathrm{N}_{b}+\mathrm{n}_{b}-\mathrm{w}_{b} \times \rho_{b} A \mathrm{v}_{b} \\
\rho_{b} \mathbf{J}_{b} \dot{\mathrm{w}}_{b}=\mathrm{M}_{b}^{\prime}+\mathrm{k} \times \mathrm{M}_{b}+\mathrm{g} \times \mathrm{N}_{b}+\mathrm{m}_{b}-\mathrm{w}_{b} \times \rho_{b} \mathbf{J}_{b} \mathrm{w}_{b} \\
\rho_{s} J_{s} \dot{\Omega}=1 / j\left(j M_{X}\right)^{\prime}+\lambda H-\beta N_{X}-\frac{\cos (\theta)}{r^{\star}} M_{\phi_{x}}+\frac{\sin (\theta)}{r^{\star}} M_{\phi_{y}}+l \\
2 \rho_{s} h_{s} \dot{V}_{x}=1 / j\left(j N_{X}\right)^{\prime}-\mu H-\frac{\cos (\theta)}{r^{\star}} N_{\phi}+f_{x}+2 \rho_{s} h_{s} \Omega V_{y} \\
2 \rho_{s} h_{s} \dot{V}_{y}=1 / j(j H)^{\prime}+\mu N_{X}+\frac{\sin (\theta)}{r^{\star}} N_{\phi}+f_{y}-2 \rho_{s} h_{s} \Omega V_{x}
\end{gathered}
$$

where $\mathbf{J}_{b}$ is equal to $\operatorname{diag}\left(I_{b}, J_{b}, J_{b}\right)$.

\subsection{Constitutive Equations}

A linear visco-elastic constitutive equation, based on the KelvinVoigt model, is chosen. In [8] and [13] we have found respectively:

$$
\begin{gathered}
\mathscr{F}_{b i}=\Sigma\left(\xi-\xi^{\star}\right)+\Upsilon(\dot{\xi}), \\
N_{X}=\frac{2 E h_{s}}{1-v^{2}}\left[\lambda\left(e_{11}+v e_{22}\right)-J_{s} \mu\left(d_{11}+v d_{22}\right)\right] \\
+\frac{6 v h_{s}}{1-v^{2}}\left[\lambda\left(\dot{e}_{11}+v \dot{e}_{22}\right)-J_{s} \mu\left(\dot{d}_{11}+v \dot{d}_{22}\right)\right] \\
N_{\phi}=\frac{2 E h_{s}}{1-v^{2}}\left[\frac{r}{r^{\star}}\left(e_{22}+v e_{11}\right)-J_{s} \frac{\sin (\theta)}{r^{\star}}\left(d_{22}+v d_{11}\right)\right] \\
\quad+\frac{6 v h_{s}}{1-v^{2}}\left[\frac{r}{r^{\star}}\left(\dot{e}_{22}+v \dot{e}_{11}\right)-J_{s} \frac{\sin (\theta)}{r^{\star}}\left(\dot{d}_{22}+v \dot{d}_{11}\right)\right] \\
H=2 h_{s} \beta\left[G+\frac{E}{1-v^{2}}\left(e_{11}+v e_{22}\right)\right]+2 h_{s} \dot{\beta}\left[v+\frac{3 v}{1-v^{2}}\left(\dot{e}_{11}+v \dot{e}_{22}\right)\right] \\
M_{X}=-\frac{2 E h_{s} J_{s}}{1-v^{2}} \lambda\left(d_{11}+v d_{22}\right)-\frac{6 v h_{s} J_{s}}{1-v^{2}} \lambda\left(\dot{d}_{11}+v \dot{d}_{22}\right) \\
M_{\phi_{x}}=-\frac{2 E h_{s} J_{s}}{1-v^{2}} \frac{r}{r^{\star}}\left(d_{22}+v d_{11}\right)-\frac{6 h_{s} J_{s}}{1-v^{2}} r^{\star} \\
M_{\phi_{y}}=0
\end{gathered}
$$

where $\Sigma(X)$ and $\Upsilon(X) \in \mathbb{R}^{6} \otimes \mathbb{R}^{6}$ are the screw stiffness matrix and the screw viscosity matrix, equal to $\Sigma=\operatorname{diag}\left(G I_{b}, E J_{b}, E J_{B}, E A, G A, G A\right), \Upsilon=v * \operatorname{diag}\left(I_{b}, 3 J_{b}, 3 J_{b}, 3 A, A, A\right)$, 
where $E$ is the Young modulus, $G$ is the shear modulus (equal to $G=E / 2(1+v)$ for an isotropic material with Poisson ratio $v$ ) and $v$ is the shear viscosity modulus.

\subsection{External Loads}

The external loads taken into account are the ones exerted by the fluid (i.e. drag, added mass, buoyancy and thrust) in addition to the gravity load. Mathematically we have:

$$
\begin{gathered}
\mathrm{n}_{b}=\mathrm{gr}_{b}+\mathrm{b}_{b}+\mathrm{d}_{b}+\mathrm{a}_{b} \\
\mathrm{n}_{s}=\mathrm{d}_{s}+\mathrm{a}_{s}+\mathrm{t}_{s}
\end{gathered}
$$

where $\operatorname{gr}_{b}(X)$ is the gravity, $\mathrm{b}_{b}(X)$ is the buoyancy, $\mathrm{t}_{s}(X)$ is the thrust load, $\mathrm{d}(X)$ is the drag and $\mathrm{a}(X)$ is the added mass.

An exhaustive derivation and interpretation of the fluid force model for the SSM is today under review, based on the usual model of net external forces exerted on a rigid rocket, uniformly "rubbed on" the mantle. Here only the final equation are reported, since it does not affect the scope of the present work. For the SRA, the fluid force models have been originally derived in [17] and then introduced in a soft robotics content in [8].

Gravity and buoyancy are simply the product between the mass per unit of $X$ of the robot arm $\rho_{b}$ and of the water $\rho_{w}$ respectively, and the gravity acceleration $g r=-9.81: \mathrm{gr}_{b}+\mathrm{b}_{b}=\left(\rho_{b}-\rho_{w}\right) A R_{b}^{T} \mathbf{G}$, where $\mathbf{G}$ is the gravity acceleration vector, equal to $\mathbf{G}=(0,0, g r)^{T}$.

The drag load vector is proportional to the square of the velocity vector and is directed in the opposite direction. The amplitude of the drag load is also determined by the geometry of section $X$ and by hydrodynamics phenomena expressed by empirical coefficients. For the SRA and the SSM respectively we have: $\mathrm{d}_{b}=-\rho_{w} \mathrm{v}_{b}^{T} \mathrm{v}_{b} D \frac{\mathrm{v}_{b}}{\left|\mathrm{v}_{b}\right|}$ and $\mathrm{d}_{s}=R_{s}^{T}\left(0,0,-\frac{\rho_{w} C_{d} A_{r e f} V|V|}{2 A_{m}}\right)^{T}$, where $D(X) \in \mathbb{R}^{3} \otimes \mathbb{R}^{3}$ is equal to $D=h_{b} * \operatorname{diag}\left(\frac{1}{2} \pi C_{x}, C_{y}, C_{z}\right)$ for circular cross sections of radius $h_{b}, C_{x}, C_{y}$, $C_{z}$ being the empirical hydrodynamic coefficients; $A_{\text {ref }}$ is the reference area equal to $\pi(\max (r(X)))^{2}, A_{m}$ is the total surface of the SSM and $C_{d}$ is the net drag coefficient. $V$ is the swimming velocity calculated at every time step as the average of the scalar field $\dot{z}(X)$, i.e. $V=\left(1 / A_{m}\right) \int_{o}^{L_{s}} \int_{0}^{2 \pi} \dot{z}\left(-z^{\star^{\prime}}\right) d X r^{\star} d \phi$.

The added mass load vector is proportional to the acceleration vector and is directed in the opposite direction. The amplitude is also determined by the geometry of section $X$ and by hydrodynamics phenomena expressed in part by correction coefficients. For the SRA and the SSM respectively we have: $\mathrm{a}_{b}=-\frac{d\left(\rho_{w} F \mathrm{v}_{b}\right)}{d t}=$ $-\rho_{w} F \dot{\mathrm{v}}_{b}-\mathrm{w}_{b} \times \rho_{w} F \mathrm{v}_{b}$ and $\mathrm{a}_{s}=-B_{s} \rho_{s} 2 h_{s} \dot{\mathrm{v}}_{s}=-B_{s} \rho_{s} 2 h_{s}\left(\dot{V}_{x}, \dot{V}_{y}, 0\right)^{T}$, where $B_{s}$ is the net added mass coefficient and $F(X) \in \mathbb{R}^{3} \otimes \mathbb{R}^{3}$ is a tensor which incorporates the geometric and hydrodynamics factors, equal to $F=\operatorname{diag}\left(0, A B_{b}, A B_{b}\right), B_{b}$ being the hydrodynamic correction coefficients. 
The thrust load is: $\mathrm{t}_{s}=R_{s}^{T}\left(0,0,-\frac{\rho_{w} \dot{U}|\dot{U}|}{A_{n} A_{m}}\right)^{T}$ where $A_{n}$ is the the nozzle area equal to $A_{n}=\pi h_{n}^{2}$ for the outflow and equal to $A_{n}=3 \pi h_{n}^{2}$ for the inflow (where three inlets and one outlet have been used with radius $h_{n}$ ) and $U$ is the mantle inner volume.

\subsection{Internal Actuation}

In order to actuate the SRA and the SSM, we impose an internal distributed wrench $\left(\mathscr{F}_{a}(X, t)\right)$ which represents the input of the model. It can be thought to be the action of the muscle fiber of the body for living organism or the result of embedded cabledriven actuation as in [8]. The final dynamics equations are as follows: $\mathscr{M}_{b} \dot{\eta}=$ $\mathscr{F}_{b i}^{\prime}+\operatorname{ad}_{\xi}^{*}\left(\mathscr{F}_{b i}\right)+\mathscr{F}_{b a}+\mathscr{F}_{b e}-\operatorname{ad}_{\eta}^{*}\left(\mathscr{M}_{b} \eta\right)$ and $\mathscr{M}_{S} \dot{\eta}=1 / j\left(j \mathscr{F}_{s i}^{1}\right)^{\prime}+\operatorname{ad}_{\xi_{\alpha}}^{*}\left(\mathscr{F}_{s i}^{\alpha}\right)+$ $\mathscr{F}_{s a}+\mathscr{F}_{s e}-\operatorname{ad}_{\eta}^{*}\left(\mathscr{M}_{s} \eta\right)$.

\section{Multi-Soft-Body Dynamic Model}

In order to model and control the behavior of a SUUV like the one in Fig. 1, a method to connect together SRAs and SSMs is needed. In this section, the link between the soft bodies in a star configuration (i.e. a tree structure with a mobile base) is shown, then the updating of the external loads of the soft bodies, due to the net motion, is discussed and finally the overall dynamics of the system is modeled.

\subsection{Star System Kinematics}

In order to link the soft bodies in a star configuration, one can pair a Gathering Rigid Body (GRB) with the already introduced frame $\left(\mathbf{e}_{1}, \mathbf{e}_{2}, \mathbf{e}_{3}\right)$. Let us call $\left(\mathbf{E}_{1}, \mathbf{E}_{2}, \mathbf{E}_{3}\right)$ the Euclidean fixed frame, hence the GRB configuration space is defined as a point $g_{r} \in S E$ (3), mapping $\left(\mathbf{E}_{1}, \mathbf{E}_{2}, \mathbf{E}_{3}\right)$ in $\left(\mathbf{e}_{1}, \mathbf{e}_{2}, \mathbf{e}_{3}\right)$ (Fig. 4), with $g_{r}=\left(\begin{array}{cc}R_{r} \mathbf{r}_{r} \\ 0 & 1\end{array}\right)$.

The time evolution of $g_{r}$ is represented by the twist vector $\eta_{r} \in \mathbb{R}^{6}$ defined by $\widehat{\eta}_{r}=g_{r}^{-1} g_{r}$. In accordance to this kinematics, the state vector of the GRB is given by the pair $\left(g_{r}, \eta_{r}\right)$ which gives immediately the kinematics equation:

$$
\dot{g}_{r}=g_{r} \widehat{\eta}_{r}
$$

At this point, the configuration of every point of the SUUV is given by $g=$ $g_{r} g_{(s, b)}$ as illustred in Fig. 4. It is worth to notice here that the number, geometry and relative position of the soft bodies can be chosen arbitrary in this scheme. As a matter of fact, in this example, we impose a rigid translation between a SSM and 
Fig. 4 illustrative scheme of the SUUV kinematics, where $\left(\mathbf{E}_{1}, \mathbf{E}_{2}, \mathbf{E}_{3}\right)$ is the Euclidean fixed frame, $\left(\mathbf{e}_{1}, \mathbf{e}_{2}, \mathbf{e}_{3}\right)$ represent the rigid body and is the soft bodies reference frame and $(\mathbf{x}, \mathbf{y}, \mathbf{z})$ is the local frame for the $\mu$ solids.

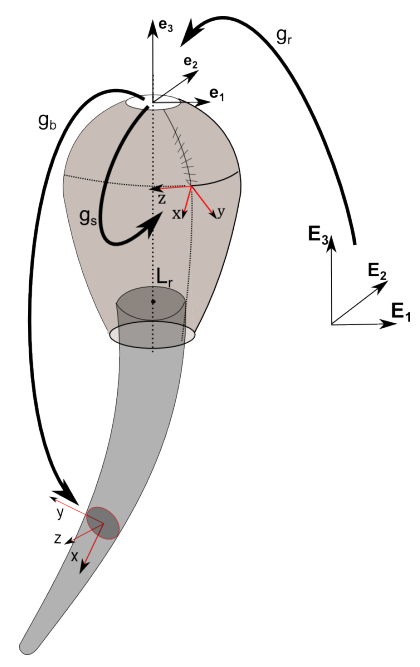

a SRA of $L_{r}$ (i.e. the length of the GRB), by adding $-L_{r}$ to the third element of $\mathbf{r}_{b}$ (Fig. 4).

\subsection{External Loads Update}

In order to take into account the overall motion, at each time step, the state vector of the GRB $\left(g_{r}, \eta_{r}\right)$ is exploited to calculate the actual value of the external loads of the soft bodies (10), (9). In our case, we have rotated the gravity vector $\mathbf{G}$ of $R_{r}^{T}$ obtaining the following new equation: $\mathrm{gr}_{b}+\mathrm{b}_{b}=\left(\rho_{b}-\rho_{w}\right) A R_{b}^{T} R_{r}^{T} \mathbf{G}$ Then, we have updated the swimming velocity $V$ and the linear velocity $\mathrm{v}_{b}$, by adding respectively the scalar $V_{r}=(0,0,0,0,0,1) \eta_{r}(i . e$. the linear velocity of the GRB in the swimming direction $\left.\mathbf{e}_{3}\right)$ and the vector $\overline{\mathrm{v}}_{r}=\operatorname{diag}(0,0,0,1,1,1) \mathrm{Ad}_{g_{b}^{-1}} \eta_{r}(i$. e. the linear velocity of the GRB transported in the local reference frame $(\mathbf{x}, \mathbf{y}, \mathbf{z}))$, where Ad is the Adjoint map. The new drag load equations for the SRA and for the SSM become: $\mathrm{d}_{b}=-\rho_{w}\left(\mathrm{v}_{b}+\overline{\mathrm{v}}_{r}\right)^{T}\left(\mathrm{v}_{b}+\overline{\mathrm{v}}_{r}\right) D \frac{\mathrm{v}_{b}+\overline{\mathrm{v}}_{r}}{\left|\mathrm{v}_{b}+\overline{\mathrm{v}}_{r}\right|}$ and $\mathrm{d}_{s}=R_{s}^{T}\left(0,0,-\frac{\rho_{w} C_{d} A_{r e f}\left(V+V_{r}\right)\left|V+V_{r}\right|}{2 A_{m}}\right)^{T}$

\subsection{Star System Dynamics}

At this point, to obtain the dynamics of the SUUV, we only miss the one of the Gathering Rigid Body that collect the soft appendices. In a geometric notation, it can be written as:

$$
\mathscr{M}_{r} \dot{\eta}_{r}=\mathscr{F}_{r}-\operatorname{ad}_{\eta_{r}}^{*}\left(\mathscr{M}_{r} \eta_{r}\right)
$$


The soft bodies of the SUUV collected together by the GRB, are frozen in their current shape. By taking advantage of that, the above unknown parameters $\left(\mathscr{M}_{r}, \mathscr{F}_{r}\right)$ can be calculated as follow ([18]): $\mathscr{M}_{r}=\int_{0}^{2 \pi} \int_{0}^{L_{s}} \operatorname{Ad}_{g_{s}}^{*} \mathscr{M}_{s} \operatorname{Ad}_{g_{s}^{-1}}\left(-z^{t^{\prime}}\right) d X r^{\star} d \phi+$ $\int_{0}^{L_{b}} \operatorname{Ad}_{g_{b}}^{*} \mathscr{M}_{b} \operatorname{Ad}_{g_{b}^{-1}} d X+\mathscr{M}_{r i}=\mathscr{M}_{s}^{\prime}+\mathscr{M}_{b}^{\prime}+\mathscr{M}_{r i}$ and $\mathscr{F}_{r}=\int_{0}^{2 \pi} \int_{0}^{L_{s}} \operatorname{Ad}_{g_{s}}^{*} \mathscr{F}_{s e}\left(-z^{\prime}\right) d X r d \phi+$ $\int_{0}^{L_{b}} \operatorname{Ad}_{g_{b}}^{*} \mathscr{F}_{b e} \sqrt{\mathrm{g}^{T} \mathrm{~g}} d X+\mathscr{F}_{r e}=\mathscr{F}_{s e}^{\prime}+\mathscr{F}_{b e}^{\prime}+\mathscr{F}_{r e}$, where $\mathscr{M}_{r i}$ and $\mathscr{F}_{r e}$ are respectively the intrinsic inertia and external load directly belonging to the GRB, and $\operatorname{Ad}_{g}^{*}=\left(\operatorname{Ad}_{g}\right)^{-T}$ is the coAdjoint map. It is worth to notice that the internal reaction and actuation of the soft bodies does not take part of these integrals, since a frozen shape have to be considered. Furthermore, as a first approximation, the inertia loads due to the ralitive acceleration of the soft bodies has not been taken into account. The contribute of the added mass loads of the soft bodies in $\mathscr{F}_{s e}^{\prime}$ and $\mathscr{F}_{b e}^{\prime}$ will appear as an additional mass as follow: $\mathscr{M}_{s a}^{\prime}=B_{s} \rho_{s} 2 h \int_{0}^{2 \pi} \int_{0}^{L_{s}} \operatorname{Ad}_{g_{s}}^{*} \operatorname{diag}(0,0,0,1,1,1) \operatorname{Ad}_{g_{s}^{-1}}\left(-z^{\star^{\prime}}\right) d X r^{\star} d \phi$ and $\mathscr{M}_{b a}^{\prime}=B_{b} \rho_{w} A \int_{0}^{L_{b}} \operatorname{Ad}_{g_{b}}^{*} \operatorname{diag}(0,0,0,0,1,1) \operatorname{Ad}_{g_{b}^{-1}} d X$.

Going forward into details, in our case, the intrinsic inertia of the GRB is equal to $\mathscr{M}_{r i}=\rho_{r} U_{r}\left(\begin{array}{cc}\operatorname{diag}\left(J_{r}, J_{r}, I_{r}\right) & \tilde{\mathbf{u}} \\ \tilde{\mathbf{u}}^{T} & \operatorname{diag}(1,1,1)\end{array}\right)$, where $\mathbf{u}=\left(0,0,-3 L_{r} / 4\right)^{T}$ is the position vector of the center of mass of the GRB whit respect to the reference frame $\left(\mathbf{e}_{1}, \mathbf{e}_{2}, \mathbf{e}_{3}\right) ; J_{r}, I_{r}$ are the second moment of inertia equal to $J_{r}=3\left(h_{r}^{2} / 4+L_{r}^{2}\right) / 5$, $I_{r}=3 h_{r}^{2} / 10$ (a conic shape have been chosen with base radius $h_{r}$ ), $\rho_{r}$ is the density and $U_{r}$ is the volume of the rigid body equal to $U_{r}=\pi h_{r}^{2} L_{r} / 3$. On the other side, the external loads on the GRB that have been considered are the gravity and buoyancy of the rigid body as well as the gravity and buoyancy of the SSM, since the letter have not been taken into account for the axisymmetric model. Thus we have: $\mathscr{F}_{r e}=\left[\left(1-\rho_{w} / \rho_{r}\right) \mathscr{M}_{r i}+\left(1-\rho_{w} / \rho_{s}\right) \mathscr{M}_{s}^{\prime}\right] \operatorname{Ad}_{g_{r}^{-1}}\left(0,0,0, \mathbf{G}^{T}\right)^{T}$.

\subsection{SUUV Dynamic Model}

The final system of equations is composed by the second order partial differential equations of the soft bodies and the ordinary differential equations of the star system. The system of p.d.e.'s is composed by the kinematics equation (2), (1), the compatibility equations (4), (3) and the dynamic equations (6), (5) respectively complemented with the internal stresses (8), (7) and the external loads (10), (9). The system of o.d.e.'s for the star system is composed by the kinematic equation (11) and the dynamic equation (12). Finally, in the state form $\dot{x}=f\left(x, x^{\prime}, x^{\prime \prime}, t\right)$, the SUUV model is: 


$$
\begin{aligned}
& \dot{\theta}=\Omega \\
& \dot{r}=\cos (\theta) V_{x}-\sin (\theta) V_{y} \\
& \dot{z}=\sin (\theta) V_{x}+\cos (\theta) V_{y} \\
& \dot{\mathbf{r}_{b}}=R_{b} \mathrm{v}_{b} \\
& \dot{R}_{b}=R_{b} \widetilde{\mathrm{w}}_{b} \\
& \dot{g}_{r}=g_{r} \widehat{\eta}_{r} \\
& \dot{\mu}=\Omega^{\prime} \\
& \dot{\lambda}=V_{x}^{\prime}+\beta \Omega-\mu V_{y} \\
& \dot{\beta}=V_{y}^{\prime}-\lambda \Omega+\mu V_{x} \\
& \dot{\mathrm{k}}=\mathrm{w}_{b}^{\prime}+\mathrm{k} \times \mathrm{w}_{b} \\
& \dot{\mathrm{g}}=\mathrm{v}_{b}^{\prime}+\mathrm{k} \times \mathrm{v}_{b}-\mathrm{w}_{b} \times \mathrm{g} \\
& \dot{\Omega}=\left[\left(j M_{X}\right)^{\prime} / j+\lambda H-\beta N_{X}-\cos (\theta) M_{\phi} / r^{\star}\right] /\left(\rho J_{s}\right) \\
& \dot{V}_{x}=\left[\left(j N_{X}\right)^{\prime} / j-\mu H-\cos (\theta) N_{\phi} / r^{\star}+2 \rho_{s} h_{s} \Omega V_{y}+f_{x}\right] /\left[2 h_{s} \rho_{s}\left(1+B_{s}\right)\right] \\
& \dot{V}_{y}=\left[(j H)^{\prime} / j+\mu N_{X}+\sin (\theta) N_{\phi} / r^{\star}-2 \rho_{s} h_{s} \Omega V_{x}+f_{y}\right] /\left[2 h_{s} \rho_{s}\left(1+B_{s}\right)\right] \\
& \dot{\mathrm{v}}_{b}=\left(\mathrm{N}_{b}^{\prime}+\mathrm{k} \times \mathrm{N}_{b}+\mathrm{n}_{b}-\mathrm{w}_{b} \times \rho_{b} A \mathrm{v}_{b}\right) /\left(\rho_{b} A\right) \\
& \dot{\mathrm{w}}_{b}=\left(\mathbf{J}_{b}^{-1} / \rho_{b}\right)\left(\mathrm{M}_{b}^{\prime}+\mathrm{k} \times \mathrm{M}_{b}+\mathrm{g} \times \mathrm{N}_{b}+\mathrm{m}_{b}-\mathrm{w}_{b} \times \rho_{b} J_{b} \mathrm{w}_{b}\right) \\
& \dot{\eta}_{r}=\mathscr{M}_{r}^{-1}\left[\mathscr{F}_{r}-\mathrm{ad}_{\eta_{r}}^{*}\left(\mathscr{M}_{r} \eta_{r}\right)\right]
\end{aligned}
$$

The final system is infinite dimensional since all its components are some functions of the profile abscissa $X$. As a result, in order to be solved numerically, it has to be first space-discretised on a grid of nodes before being time integrated using explicit or implicit time integrators starting from the initial state. In this grid, all the space derivatives appearing in the p.d.e.'s system can be approximated by finite difference schemes, with the following boundary conditions: $\eta(0)=0$ and $\mathscr{F}_{b i}\left(L_{b}\right)=\mathscr{F}_{s i}^{1}\left(L_{s}\right)=0$. These operations have been implemented in Matlab ${ }^{\complement}$.

\section{Results}

Although the final goal of this work is to model and control SUUVs like the one in Fig. 1, whereby experimental comparison are needed (as has been done separately for the SRA [8] and for the SSM [14]), in this section an illustrative example, based on simulation, of the setting developed above is presented, in order to demonstrate the feasibility of the proposed mathematical framework to achieve the objective. Further simulation analysis and experimental comparisons with the real prototype, are planned for the extension of the present paper. Finally, an energetic analysis disclosed by the current formulation is conducted and used to describe the results.

\subsection{Simulation}

One SRA and one SSM have been used, the former has a conical shape with a radius linearly decreasing from $\max \left(h_{b}(X)\right)=15$ to $\min \left(h_{b}(X)\right)=6 \mathrm{~mm}$, the latter 
is a semi-sphere of radius $31 \mathrm{~mm}$ glued with a cylinder of length $86 \mathrm{~mm}$, both with an half thikness of $h_{s}=1 \mathrm{~mm}$. The GRB has a conical shape too, with a base radius equal to $h_{r}=\max \left(h_{b}(X)\right)=15 \mathrm{~mm}$ and an height of $L_{r}=112 \mathrm{~mm}$. The density of these bodies has been chosen equal to the one of the water $\left(1022\left[\mathrm{~kg} / \mathrm{m}^{3}\right]\right)$, which makes the structure neutral underwater. The geometrical and mechanical parameters are summarized in Table 1.

Table 1 Geometrical and mechanical parameters of the SUUV.

\begin{tabular}{llllllll}
\hline & $E[\mathrm{kPa}]$ & $v[\mathrm{~Pa} * \mathrm{~s}]$ & $v[-]$ & $\rho\left[\mathrm{kg} / \mathrm{m}^{3}\right]$ & $L[\mathrm{~mm}]$ & $h[\mathrm{~mm}]$ & $h_{n}[\mathrm{~mm}]$ \\
\hline SSM & 40 & 500 & 0 & 1022 & 130 & 1 & 10 \\
SRA & 110 & 300 & 0 & 1022 & 420 & {$[6,15]$} & - \\
GRB & - & - & - & 1022 & 112 & 15 & - \\
\hline
\end{tabular}

In order to reproduce the jet propulsion of the mantle, the SSM is actuated through a triangular wave force function $f_{s}(X, t)$, perpendicular to the axis of symmetry (thus along $\mathbf{e}_{r}$ ), with period $T$ and amplitude ranging in the interval $\left[F_{\min }, F_{\max }\right]$. This pressure has been applied to a central strip of the mantle of height $80 \mathrm{~mm}$. to reproduce the bending/steering capability of the robot arm, the SRA is actuated through a linear torque function $f_{b}(X, t)$ with extremes $\left[M_{\min }, M_{\max }\right]$, directed toward the local axis $\mathbf{z}$ for a certain interval $\Delta t_{1}$ and toward the direction $\mathbf{y}$ for another interval $\Delta t_{3}$, preceded and followed by a rest period of respectively $\Delta t_{2}$ and $\Delta t_{4}$. In other words, the internal distributed wrench $\mathscr{F}_{s a}(X, t)$ takes the form: $\mathscr{F}_{s a}=$ $\operatorname{Ad}_{g_{3}^{-1}}^{*}\left(0,0,0, f_{s}, 0,0\right)^{T}$, and the internal distributed wrench $\mathscr{F}_{b a}(X, t)$ takes different forms for each interval $\Delta t_{1}, \Delta t_{2}, \Delta t_{3}, \Delta t_{4}$, respectively: $\mathscr{F}_{b a}=\left(0,0, f_{b}, 0,0,0\right)^{T}$, $\mathscr{F}_{b a}=(0,0,0,0,0,0)^{T}, \mathscr{F}_{b a}=\left(0, f_{b}, 0,0,0,0\right)^{T}, \mathscr{F}_{b a}=(0,0,0,0,0,0)^{T}$. The loading and dynamic parameters are summarized in Table 2, while a few snapshots of the resulting swimming dynamics is depicted in Figure 5.

Table 2 Loading and dynamic parameters of the SUUV.

\begin{tabular}{lllllll}
\hline & {$\left[F_{\text {min }}, F_{\text {max }}\right]$} & $T[s]$ & {$\left[M_{\text {min }}, M_{\text {max }}\right]$} & $\left(\Delta t_{1}, \Delta t_{2}, \Delta t_{3}, \Delta t_{4}\right) B[-]$ & $C_{d}$ & $C_{(x, y, z)}[-]$ \\
{$[P a]$} & & {$[N]$} & {$[s]$} & & {$[-]$} & \\
\hline SSM $[0,5]$ & 0.66 & - & - & 1.1 & 1.7 & - \\
SRA - & - & {$[0,0.05]$} & $(1.5,3,1.5,3)$ & 1.5 & - & $(0.01,1.5,1.5)$ \\
\hline
\end{tabular}

Although a simple actuation pattern has been applied, a complex swimming dynamics came out from the fluid-structure interaction, with unexpected turning around the symmetry axis of the shell mantle $\left(\mathbf{e}_{3}\right)$ due to a torsional torque occurred during the switching from one bending to the other. This represents a further motivation toward the development of a proper model of the SUUVs dynamics, in order 
Fig. 5 Few snapshots of the simulation results at $t=0$, $t=1.5, t=4, t=5.5, t=7.5$ and $t=9 \mathrm{~s}$ of the swimming dynamics. The complete video is presented in the media file attached to this paper.

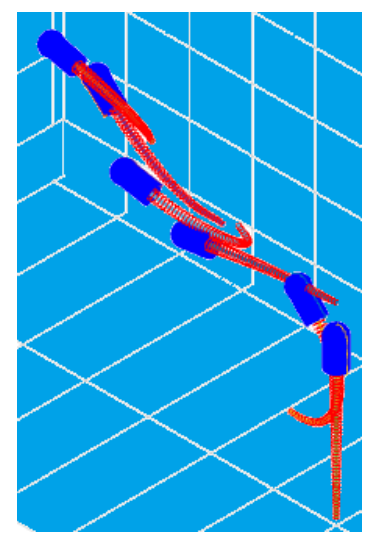

to be able to understand, design and control these promising devices for underwater exploration.

\subsection{Energetic Analysis}

Any kind of locomotion is the result of the dynamic interaction between the body deformation and the environment [19]. The quality of this interaction can be measured by calculating how the internal actuation power is translated into the kinetic power of the star system. In the present work, the internal actuation power have been approximate with the positive part of the stress power, reflecting that the elastic energy is mainly due to this action and that it can be only increased by the internal actuation here. With this quantitative index, one can play with the geometrical, mechanical and actuation parameters during the design phase in order to find the best solution for a given application. This is probably one of the killer application of the model.

To do so, we resort to the following efficiency index computed at each time step throughout the simulation $E(t): E=\frac{W_{o}}{W_{i}}$, where $W_{o}(t)$ and $W_{i}(t)$ respectively represent the output kinetic power of the star system and the input actuation power. Calculating them we obtain: $W_{o}=\eta_{r}^{T} \mathrm{M}_{r} \dot{\eta}_{r}$ and $W_{i}=W_{i s}+W_{i b}=\int_{0}^{L_{s}} \int_{0}^{2 \pi}\left\{\frac{2 E h_{s}}{1-v^{2}}\left[E_{11} \dot{E}_{11}+\right.\right.$ $\left.\left.E_{22} \dot{E}_{22}+J_{s}\left(D_{11} \dot{D}_{11}+D_{22} \dot{D}_{22}\right)\right]+2 h_{s} G \beta \dot{\beta}\right\}\left(-z^{\prime}\right) d X r d \phi+\int_{0}^{L_{b}}\left(\xi-\xi^{\star}\right)^{T} \Sigma \dot{\xi} \sqrt{g^{T} g} d X$, where $W_{i s}(t)$ and $W_{i b}(t)$ are respectively the actuation contribution of the SSM and of the SRA (equal to the positive part of the internal elastic energy and to zero in the other case) and we have defined $E_{11}=e_{11}+v e_{22}, E_{22}=e_{22}+v e_{11}$, $D_{11}=d_{11}+v d_{22}$ and $D_{22}=d_{22}+v d_{11}$. In Fig. 6, the actuation and kinetic power corresponding to the simulation of Fig. 5 are shown. The mean value of the index $E$ is around $3 \%$. 
Fig. 6 Kinematic (blu) and actuation (red) power (note the different scales). The four phases of the simulation of Figure 5 are highlighted.

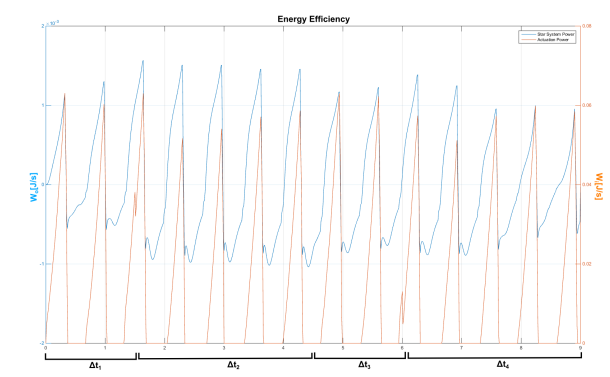

In Figure 6 can be recognized the different phases of the motion of Fig. 5. In the first part $\left(\Delta t_{1}\right)$, the input power is composed by the cyclic contraction of the SSM and by the bending of the SRA. In the second phase $\left(\Delta t_{2}\right)$, the output kinetic power benefits from the released elastic energy of the SRA and from the positive asset of the new configuration, giving a larger gap with respect to the actuation power. In the third part $\left(\Delta t_{3}\right)$, the actuation power increase due to the new bending and in the last phase $\left(\Delta t_{4}\right)$ the SUUV start to turn around it self due to the hydrodynamic coupling of the bent SRA which is not as efficient as the forward pushing of the second phase, giving a smaller gap between the kinetic and actuation power.

\section{Conclusion}

A unified model which account for the continuum nature of a multi-soft-body robot has been presented which allows to describe the dynamics of an underwater vehicle while it travels in a quiescent fluid. The potentialities of the model have been demonstrated through an illustrative example, which shows complex and unexpected dynamics of the robot despite of a regular actuation input, underlining how much the behavior of this new kind of systems can be rich and challenging to control. Furthermore, an energetic analysis which takes into account the internal actuation and the mechanical properties of the vehicle is proposed. The work presented here represents a first step toward the development of a mathematical tool for the design and control of intelligent SUUVs.

Acknowledgements This work was partly supported by the Foundation Grant project PoseiDRONE of the Fondazione Livorno.

\section{References}

1. S. Saimek and Perry Y. Li "Motion Planning and Control of a Swimming Machine" International Journal of Robotics Research, vol. 23, pp. 27-53, 2004. 
2. J. E. Colgate and K. M. Lynch "Mechanics and control of swimming: a review" IEEE Journal of Oceanic Engineering, vol. 29, pp. 660-673, 2004.

3. A. D. Marchese, C. D. Onal and D. Rus "Autonomous Soft Robotic Fish Capable of Escape Maneuvers Using Fluidic Elastomer Actuators" Soft Robotics, vol. 1, pp. 75-87, 2014.

4. B.A. Trimmer "Soft Robots" Current Biology, vol. 23, pp. 639-641, 2013.

5. A. Arienti, M. Calisti, F. Giorgio-Serchi and C. Laschi "PoseiDRONE: design of a soft-bodied ROV with crawling, swimming and manipulation ability" Proceedings of the MTS/IEEE OCEANS conference, San Diego, CA, USA, September, 21-27, 2013.

6. F. Giorgio-Serchi, A. Arienti, I. Baldoli and C. Laschi "An elastic pulsed-jet thruster for Soft Unmanned Underwater Vehicles" Robotics and Automation (ICRA), 2013 IEEE International Conference on, pp.5103,5110, 6-10 May 2013.

7. F. Giorgio-Serchi, A. Arienti and C. Laschi "A soft unamnned underwater vehicle with augmented thrust capability" Proceedings of the MTS/IEEE OCEANS conference, San Diego, CA, USA, September, 21-27, 2103.

8. F. Renda, M. Giorelli, M. Calisti, M. Cianchetti, C. Laschi, 'Dynamic Model of a Multibending Soft Robot Arm Driven by Cables" Robotics, IEEE Transactions on, Vol 30, no 5, pp. 1109-1122, 2014.

9. M. Calisti, M. Giorelli, G. Levy, B. Mazzolai, B. Hochner, C. Laschi and P. Dario "An octopus-bioinspired solution to movement and manipulation for soft robots" Bioinspiration and Biomimetics, vol. 6, pp. 1-10, 2011.

10. M. Giorelli, F. Giorgio-Serchi, A. Arienti and C. Laschi "Forward speed control of a pulsedjet soft-bodied underwater vehicle" Proceedings of the MTS/IEEE OCEANS conference, San Diego, CA, USA, September, 21-27, 2013.

11. J. C. Simo "A finite strain beam formulation. The three dimensional dynamic problem: part I" Comput. Methods Appl. Mech. Eng., vol. 49, pp. 55-70, 1985.

12. J. C. Simo and D. D. Fox, "On stress resultant geometrically exact shell model. Part I: formulation and optimal parametrization" Journal Computer Methods in Applied Mechanics and Engineering vol. 72, no. 3, pp. 267-304, Mar 1989.

13. F. Renda, F. Giorgio-Serchi, F. Boyer and C. Laschi "Structural Dynamics of a Pulsed-Jet Propulsion System for Underwater Soft Robots" Int J Adv Robot Syst, vol. 12, no. 68, 2015.

14. F. Renda, F. Giorgio-Serchi, F. Boyer and C. Laschi "Locomotion and Elastodynamics Model of an Underwater Shell-like Soft Robot" Robotics and Automation (ICRA), 2015 IEEE International Conference on, pp.1158,1165, 26-30 May 2015, Seattle, USA, May 26-30, 2015.

15. F. Giorgio-Serchi, F. Renda, M. Calisti and C. Laschi, "Thrust depletion at high pulsation frequencies in underactuated, soft-bodied, pulsed-jet vehicles ", MTS/IEEE OCEANS, Genoa, Italy, May 19-21, 2015.

16. S. S. Antman, Nonlinear Problems of Elasticity, 2nd edn (Applied Mathematical Sciences vol 107) (New York:Springer), 2005.

17. F. Boyer, M. Porez and W. Khalil "Macro-Continuous Computed Torque Algorithm for a Three-Dimensional Eel-Like Robot" Robotics, IEEE Transactions on, vol. 22, no.4, pp. 763 775, 2006.

18. F. Boyer, S. Ali and M. Porez "Macrocontinuous Dynamics for Hyperredundant Robots: Application to Kinematic Locomotion Bioinspired by Elongated Body Animals" Robotics, IEEE Transactions on, vol. 28, no.2, pp. 303 317, April 2012.

19. F. Boyer and A. Belkhiri "Reduced locomotion dynamics with passive internal DoF: application to nonholonomic and soft robotics" Robotics, IEEE Transactions on, vol.30, no.3, pp.578,592, June 2014 . 